\title{
SILI functions as an oncogene in glioma by AKT/mTOR signaling pathway
}

This article was published in the following Dove Press journal:

OncoTargets and Therapy

\author{
Hao Xu \\ Shangchen $\mathrm{Xu}$ \\ Rui Zhang \\ Tao Xin \\ Qi Pang
}

Department of Neurosurgery, Shandong Provincial Hospital Affiliated to Shandong University, Jinan 25002I, Shandong, China
Correspondence: Tao Xin; Qi Pang Department of Neurosurgery, Shandong Provincial Hospital Affiliated to Shandong University, No. 324, JingWu Road, Jinan 25002I, Shandong, China

Tel/fax +86 53I 879389। I

Email dr_xintao@sina.com; dr_pangqi@sina.com
Purpose: SIL1 is a ubiquitous protein localized to the endoplasmic reticulum and functions as a cochaperone of BiP. Previous studies have shown that function loss of SIL1 is often associated with neurological diseases, such as Marinesco-Sjögren Syndrome. However, no studies have investigated the function of SIL1 in tumors. In this study we aim to reveal functions of SIL1 and the underlying mechanisms in glioma.

Materials and methods: First, by searching on Gene Expression Profiling Interactive Analysis, we examined SIL1 expression and prognostic value in glioblastoma multiforme (GBM) and brain lower grade glioma (LGG). Immunohistochemical analysis (IHC) was also performed to determine the endogenic SIL1 level. Cell counting kit-8 (CCK8) and clone formation assays were used to detect cell proliferation of U251 cells. Cell migration was detected by transwell assay and cell cycle and apoptosis were detected by flow cytometry. Western blot was performed to determine protein expression.

Results: We found that the expression of SIL1 was increased by approximately 1.5 -fold in GBM and 1.3-fold in LGG compared with normal controls $(P<0.05)$ and negatively correlated with patients' survival. IHC revealed that SIL1 expression was significantly higher in glioma tissues than that in paracancerous tissues $(P<0.05)$. Glioma patients with high SIL1 expression accounted for $65.79 \%(25 / 38)$ of total samples and SIL1 expression significantly increased in grade IV glioma compared to grades I-III ( $P=0.026)$. Suppression of SIL1 expression led to significant inhibition of $\mathrm{U} 251$ cell proliferation. Transwell assay showed that cell migration of U251 was significantly inhibited by siSIL transfection, with an inhibitory rate reaching $69 \%$. Flow cytometry detection showed that siSIL1 could induce apoptosis of U251 cells and upregulated the expression of the pro-apoptotic protein Bax and Caspase3-P17. However, siSIL1 transfection had no effect on the cell cycle. Mechanism studies demonstrated that siSIL1 transfection led to inactivation of AKT/ mTOR signaling pathway, including decreased phosphorylation of AKT and mTOR without affecting protein expression, as well as decreased expression of the downstream effector p70S6K.

Conclusion: Downregulation of SIL1 inhibited the progression of glioma by suppressing the AKT/mTOR signaling pathway.

Keywords: SIL1, tumor, prognosis, proliferation, migration, apoptosis

\section{Introduction}

Gliomas are the most common tumors in the central nervous system, accounting for about $45 \%$ of all intracranial tumors. ${ }^{1}$ In the past 30 years, the incidence of primary malignant brain tumors increased with an annual growth rate of about $1.2 \% .^{2}$ Despite great improvement in surgical resection, chemotherapy and radiotherapy, patients suffering from gliomas still have a poor survival, usually 2-6 years. ${ }^{3}$ Glioblastoma, ie, grade IV glioma, is the most aggressive and life-threatening glioma tumor. Medical survival of patients with glioblastoma ranges from 12 to 16 months. ${ }^{4}$ Due to high 
mortality of glioma patients, it is urgent to explore new biomarkers or molecular targets for the prognosis and therapy of glioma patients.

Originally found in yeast, SIL1 functions as a cochaperone of BiP, which is an endoplasmic reticulum (ER) located HSP70 protein participating in protein translocation, folding and ER stress sensing. ${ }^{5} \mathrm{BiP}$ function depends on the hydrolysis of the combined ATP and ADP/ATP exchange. ${ }^{6}$ SIL1, also called BiP-associated protein (BAP), functions as a nucleotide exchange factor for BiP to regulate its activity. ${ }^{6,7}$ BiP and SIL1 are ubiquitously expressed in human tissues and their abnormal expression or structure disruption often leads to cell protein abnormal accumulation, impaired ER homeostasis and even cell apoptosis. ${ }^{8-11}$ However, it is interesting that function loss of SIL1 causes a selective vulnerability of certain tissues, especially the nervous system and skeletal muscle. Moreover, SIL1 is revealed to exert a neuroprotective action in neurodegenerative and neuromuscular disorders, such as amyotrophic lateral sclerosis (ALS), ${ }^{12}$ Alzheimer's disease (AD), ${ }^{9}$ and Marinesco-Sjögren syndrome (MSS). ${ }^{10,11,13}$ Filézac de L'Etang et al find that in an ALS mouse model SIL1 protein exhibits a differential expression in different motor neurons and knocking out of Sil1 disrupts ER homeostasis and aggravates the degree of ALS. ${ }^{12}$ Liu et al report that SIL1 expression is decreased in AD mouse model and overexpression of SIL1 prominently reduces BiP elevationrelated tau hyperphosphorylation and GSK-3 $\beta$ activation in ER stress. ${ }^{9}$ Howes et al find that $\mathrm{C}$-terminal mutations disrupt SIL1 structural integrity and lead to MSS. ${ }^{11}$ Due to the close correlation with neurological function, SIL1 may play a role in nervous system tumors such as glioma. So far there are few studies exploring the expression of SIL1 in glioma and its role in the tumor pathobiology.

Here we investigated SIL1 expression and its prognostic relevance in glioma. To explore the physiological action of SIL1, we applied RNA interference technology to knock down the SIL1 expression in U251 glioma cells and investigated the impact on tumor cell function.

\section{Materials and methods Materials}

Dulbecco's Modified Eagle's Medium (DMEM) was purchased from HyClone (Thermo Fisher Scientific, Waltham, MA, USA). Fetal bovine serum (FBS) was purchased from Thermo Fisher Scientific. Penicillin-streptomycin, 0.25\% trypsin and cell counting kit-8 (CCK8) agent were purchased from Beijing Solarbio Science \& Technology Company (Beijing, China). Lipofectamine 2000 was purchased from
Thermo Fisher Scientific. Annexin V-fluorescein isothiocyanate (FITC)/propidium iodide (PI) Apoptosis Kit was purchased from Thermo Fisher Scientific. Radioimmunoprecipitation assay (RIPA) lysis buffer, bicinchoninic acid (BCA) protein assay kit, protease inhibitor cocktail, and all antibodies were purchased from CWBIO (Beijing, China). Primers were synthesized by Genewiz Company (Beijing, China). Transwell cell culture plates were purchased from EMD Millipore (Billerica, MA, USA). Protein marker was purchased from Thermo Fisher Scientific. Electrochemi luminescence (ECL) developer was purchased from Proteintech Group (Chicago, IL, USA).

\section{Immunohistochemical assay}

Immunohistochemical (IHC) analysis was performed by using an EliVision ${ }^{\text {TM }}$ Plus Kit (KIT-9902; Boster Biological Technology, Pleasanton, CA, USA). The tissue sections were obtained from Shandong Province Hospital. This work has been approved by the ethical committees at Shandong Provincial Hospital. All patients provided written informed consent. Glioma tumor or paracancerous tissues were cryosectioned at $8 \mu \mathrm{m}$ thickness. Sodium citrate buffer ( $\mathrm{pH}$ 6.0) was used for antigen retrieval. Slides were incubated with $3 \% \mathrm{H}_{2} \mathrm{O}_{2}$ to clear endogenous peroxidase. Then the sections were blocked for $1 \mathrm{~h}$ at room temperature and incubated with primary antibodies. 3,3'-diaminobenzidine tetrahydrochloride (DAB) developer was used for slide development. After doubled staining with hematoxylin, dehydration with gradient ethanol, and mounting with neutral resins, tissue sections were observed under a microscope.

Under the $40 \times$ objective, 3-5 viewing fields were randomly chosen. The staining was scored as " 0 " (no staining), "1" (weakly positive), "2" (moderately positive), and " 3 " (strongly positive). The percentage of positively stained cells was scored as: $0=0 \%, 1=1 \%-25 \%, 2=26 \%-50 \%$, $3=51 \%-75 \%$, and $4=76 \%-100 \%$. Two independent pathologists were involved in the evaluation of slides. Low expression was identified when calculation of the score was less than 6 , the cut-off derived from X-tile analysis, ${ }^{14}$ otherwise they were defined as high expression.

\section{Cell culture and transfection}

U251 glioma cells were purchased from the Cell Bank of the Chinese Academy of Sciences (Shanghai, China). Cells were cultured in DMEM medium added with $10 \% \mathrm{FBS}$, $100 \mathrm{U} / \mathrm{mL}$ penicillin, and $0.1 \mathrm{mg} / \mathrm{mL}$ streptomycin at $37^{\circ} \mathrm{C}$ in a humidified atmosphere containing $5 \% \mathrm{CO}_{2}$. U251 cells were digested when entered in logarithmic phase and then planted 
into a 6-well plate. siRNA for SIL1 was transfected into U251 cells using Lipofectamine 2000 following the instructions. A scrambled RNA was used as negative control in all experiments. siSIL1 sequence was as follows:

siRNA1: GGCUGGAUAUCAACACCAATT UUGGUG

UUGAUAUCCAGCCTT

siRNA2: CGGAGAAGAUGUUCGCCGATT UCGG

CGAACAUCUUCUCCGTT

siRNA3: UGGUACGGCUGAUCAACAATT UUGU

UGAUCAGCCGUACCATT

siRNA4: CGCUCACUGCAAAGAAGAATT UUCUU CUUUGCAGUGAGCGTT

Primer sequence, upstream: 5'-TGCTTCACCTT

CTGCCTCAG-3'; downstream: 5'-GAACACCTCCA GGACTTCGG-3'.

\section{Fluorescence quantitative PCR}

Ultrapure RNA extraction kit was used to extract total RNA of siSIL1/siNC transfected U251 cells. Total RNA was then reverse transcribed to cDNA using a PrimeScript RT Master Mix kit (Takara, Tokyo, Japan) according to the manufacturer's protocol. Amplifications were performed in a FTC-3000 Real-Time Quantitative Thermal Cycler (Funglyn Biotech Inc., Shanghai, China). The gene expression data were analyzed using the $2-\Delta \Delta \mathrm{Ct}$ method.

\section{CCK8 assay}

Cell viability was detected by using CCK8 (Solarbio Biotech Corp., Beijing, China) following the manufacturer's instructions. Briefly, $1-5 \times 10^{3} \mathrm{U} 251$ cells were planted into each well of a 96-well plate. At 24, 48, 72, and $96 \mathrm{~h}$ after transfection, $10 \mu \mathrm{L} \mathrm{CCK} 8$ solution was added and incubated for $2 \mathrm{~h}$. The cell viability was determined by measuring OD value at $450 \mathrm{~nm}$.

\section{Clone formation assay}

After transfection for $24 \mathrm{~h}, 200$ cells were planted in a $6 \mathrm{~cm}$ dish with $5 \mathrm{~mL}$ medium. Sustained culturing was performed until the clones could be visible in naked eyes. Then remove the medium and stain the clones with $0.1 \%$ crystal violet. The clones were imaged and counted.

\section{Cell migration assays}

U251 cells transfected with siSIL1 or siNC were digested and planted in a 24-well transwell insert, the pore size of which was $8.0 \mu \mathrm{m}$. $500 \mu \mathrm{L}$ DMEM (FBS, 10\%) was added to the lower chamber. After incubation for $48 \mathrm{~h}$, the residual cells on the top surface of membrane were removed by scraping and migrated cells were fixed by $4 \%$ paraformaldehyde for
$30 \mathrm{~min}$, and then stained with $0.1 \%$ crystal violet for $20 \mathrm{~min}$. Migrated cells were quantified by counting in five separated visual fields.

\section{Flow cytometry for apoptosis detection}

Cell apoptosis was analyzed using FITC/PI Apoptosis Kit (Thermo Fisher Scientific). After transfection for 48 h, U251 cells were collected and washed with precooled PBS. Then cells were centrifuged and the supernatant was carefully removed. Annexin V binding buffer was added to resuspend cells to $1-5 \times 10^{6} / \mathrm{mL}$. Cell suspension $(100 \mu \mathrm{L})$ was incubated with $5 \mu \mathrm{L}$ Annexin V/FITC mix for $5 \mathrm{~min}$. Then $10 \mu \mathrm{L}$ PI dye and $400 \mu \mathrm{L}$ PBS were added before flow cytometry. Statistical analysis was performed using Flowjo software.

\section{Western blot}

After transfection for $48 \mathrm{~h}$, total cell proteins were extracted and separated by sodium dodecyl sulfate polyacrylamide gel electrophoresis (SDS-PAGE). Then protein bands were transferred onto a polyvinylidene fluoride (PVDF) membrane. After blocking with 5\% non-fat milk for $1 \mathrm{~h}$, the membrane was incubated at $4^{\circ} \mathrm{C}$ overnight with primary antibodies and second antibodies for $1 \mathrm{~h}$ at room temperature. The protein bands were developed by ECL system and quantified with QUANTITY ONE software. The primary antibodies included anti-SIL1 (Cat\#ab5639; Abcam, Cambridge, UK), anti- $\alpha$ tubulin (Cat\#ab18251; Abcam), anti-Bax (Cat\#ab32503; Abcam), anti-active caspase3 p17-specific (Cat\#25546-1-AP; Proteintech Group), anti-p-Akt (Cat\#ab81283; Abcam), anti-Akt (Cat\#ab32505; Abcam), anti-mTOR (Cat\#ab2732; Abcam), anti-p-mTOR (Cat\#ab131538; Abcam), antiP70S6K (Cat\#ab32529; Abcam). The secondary antibodies were purchased from Proteintech.

\section{Statistical analysis}

All data were represented from three independent experiments and expressed as mean \pm standard deviation. SPSS 18.0 software (version 16.0; SPSS Inc., Chicago, IL, USA) was used for all the statistical analysis. The differences between two groups were analyzed using Student's $t$-test. $P<0.05$ was considered statistically significant.

\section{Results}

\section{SILI expression in glioma}

Recent studies revealed that elevation of SIL1 expression could play a neuroprotective effect in ALS, AD, or MSS. ${ }^{9,10,12}$ In order to investigate the function of SIL1 in glioma, which is 
also a nervous system related disease, we checked mRNA expression of SIL1 on Gene Expression Profiling Interactive Analysis (GEPIA), an online server derived from the Cancer Genome Atlas (TCGA), and Genotype-Tissue Expression (GTEx) dataset. ${ }^{15}$ The box plots shown in Figure 1A revealed that SIL1 expression significantly increased in glioblastoma multiforme (GBM) by about 1.5 fold and brain lower grade glioma (LGG) by about 1.3 fold compared to normal controls $(P<0.05)$. The result suggested that SIL1 might be involved in glioma progression.

\section{High SILI expression represented a poor prognosis}

GEPIA also provided survival analysis, which revealed the correlation between gene expression and the survival rate of patients, so as to describe the prognostic value of a specific gene. The survival curves of GBM and LGG patients with high or low SIL1 expression were shown in Figure 1B. The results indicated that the survival rate of patients with a high SIL1 expression was significantly lower in both GBM $(\mathrm{HR}($ high $)=1.7, p(\mathrm{HR})=0.0053, \mathrm{n}($ high $)=81, \mathrm{n}(\mathrm{low})=81)$ and
A

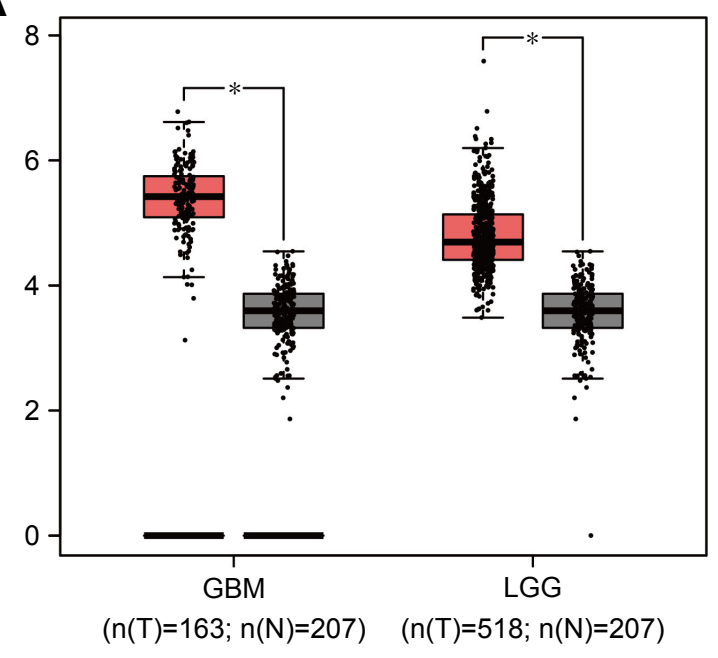

B

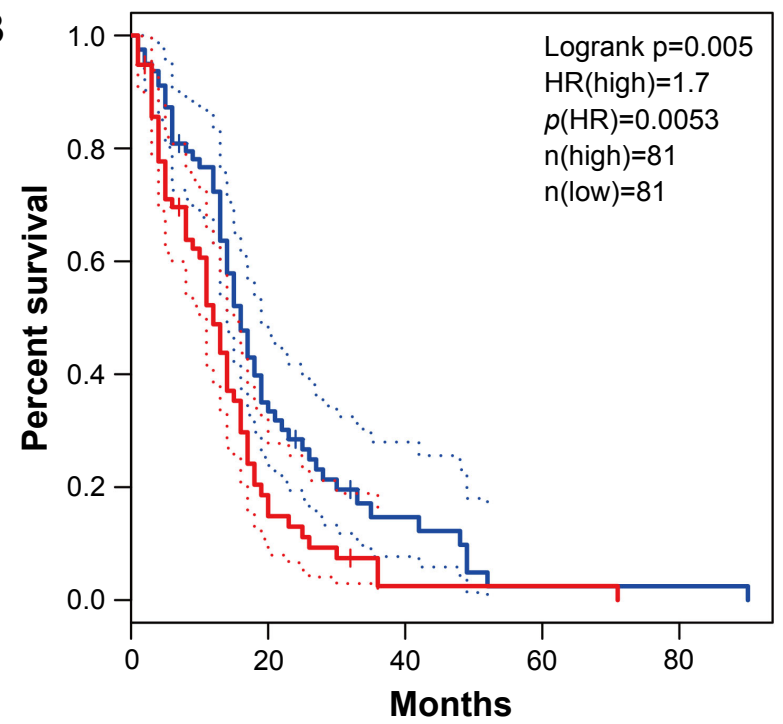

C
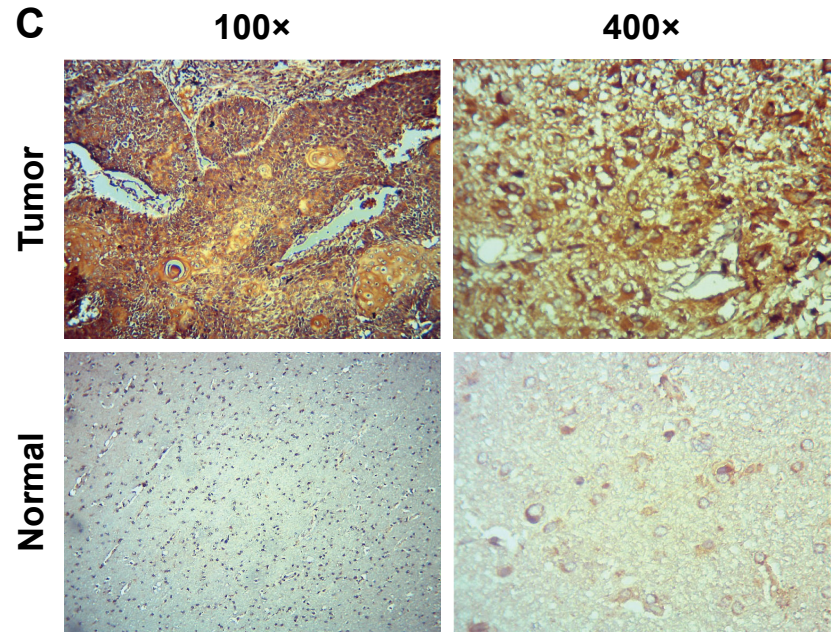

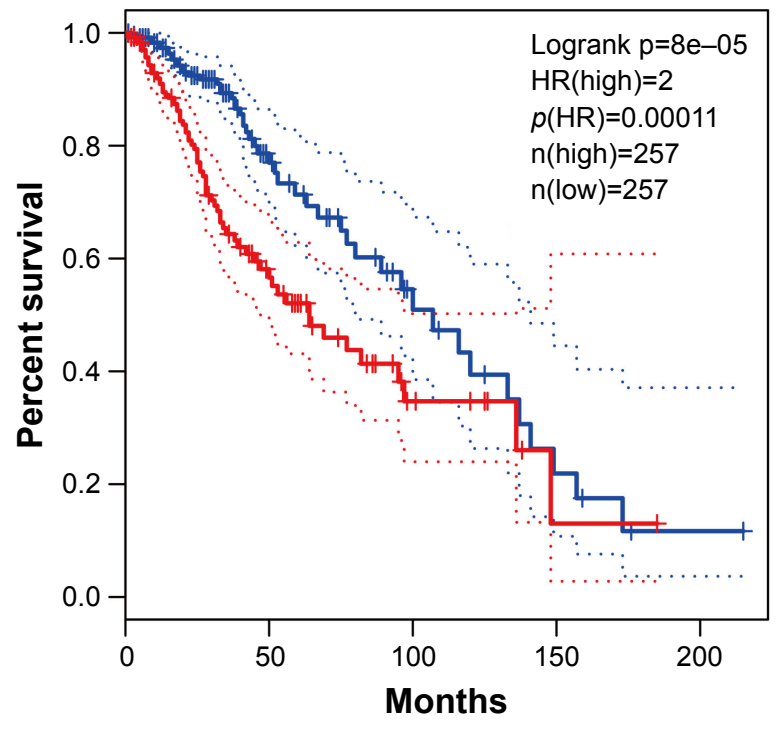

Low SIL1 TPM — High SIL1 TPM

Figure I SILI was highly expressed in glioma and represented a poor prognosis. (A) The red and gray boxes represent glioma and normal tissues, respectively. The $y$-axis indicates the log2-transformed gene expression level. (B) Survival percentage of glioma patients with high or low SILI expression. Left: GBM; right: LGG. The data of (A) and (B) were obtained with permission from, Tang Z, et al. (20I7) GEPIA: a web server for cancer and normal gene expression profiling and interactive analyses. Nucleic Acids Res. 10.1093/nar/gkx247. ${ }^{15}$ (C) Endogenous levels of SILI in glioma and paracarcinoma tissues were tested by immunohistochemical assay. Note: $* p<0.05$.

Abbreviations: GBM, glioblastoma multiforme; GEPIA, Gene Expression Profiling Interactive Analysis; LGG, lower grade glioma; TCGA, The Cancer Genome Atlas; HR, hazards ratio; TPM, trans per million. 
$\mathrm{LGG}(\mathrm{HR}($ high $)=2, p(\mathrm{HR})=0.00011, \mathrm{n}($ high $)=257, \mathrm{n}($ low $)=257)$ compared to those with a low SIL1 expression. It was suggested that high SIL1 expression represented a poor prognosis and SIL1 might play a role in promoting glioma progression.

In order to acquire more intuitive understanding of endogenous SIL1 level, we performed IHC staining in glioma tissue and paracarcinoma tissue derived from the same patient. Results in Figure 1C revealed a strong immunohistochemistry staining of SIL1 in glioma tissue, while that was very weak in normal tissue. It was demonstrated that SIL1 expression was significantly enhanced in glioma tissue, which was consistent with the result in box plots. In addition, SIL1 expression and its correlation with clinical pathological features of glioma patients was also investigated (Table 1). In general, glioma patients with high SIL1 expression accounted for $65.79 \%$ $(25 / 38)$ of total samples. SIL1 expression had no connection with age or sex of patients but significantly increased in grade IV glioma ( $P=0.026)$ compared to grades I-III.

\section{Downregulation of SILI inhibited U25 I cell proliferation and migration}

To investigate the function of SIL1, we designed 4 siRNAs to knock down SIL1 in U251 cells. The interference efficiencies were quantified and plotted in Figure 2A. The result indicated that among the four kinds of siRNAs, siRNA2 and siRNA3 exhibited the most powerful activity in knocking down SIL1 expression. So we selected siRNA3 (siSIL1) in the follow-up experiments. The Western blot result in Figure 2B verified the inhibition of siSIL1 on SIL1 expression.

Sustaining proliferation was the most important property of cancer cells, so we first detected the effect of SIL1 downregulation on U251 cell proliferation by using CCK8 assay and clone formation assay. The result shown in Figure 2C

Table I SILI expression and the relationship between clinical pathological features of glioma patients

\begin{tabular}{lllll}
\hline $\begin{array}{l}\text { Clinical } \\
\text { pathological } \\
\text { features }\end{array}$ & $\begin{array}{l}\text { Cases } \\
\text { (n) }\end{array}$ & \begin{tabular}{l} 
SILI level \\
\cline { 3 - 4 }
\end{tabular} & $\begin{array}{l}\text { High } \\
\text { expression (n) }\end{array}$ & $\begin{array}{l}\text { Low } \\
\text { expression (n) }\end{array}$ \\
\hline $\begin{array}{lllll}\text { Age } \\
\quad 60\end{array}$ & 10 & 6 & 4 & 0.653 \\
$>60$ & 28 & 19 & 9 & \\
$\begin{array}{l}\text { Sex } \\
\quad \text { Male }\end{array}$ & 19 & 11 & 8 & 0.739 \\
$\quad \begin{array}{l}\text { Female } \\
\text { Pathological grade* }\end{array}$ & 19 & 12 & 7 & \\
I-III & 28 & 14 & 14 & \\
IV & 10 & 9 & 1 & \\
\hline
\end{tabular}

Note: $*$ Significant difference, $P<0.05$. indicated that after transfection for $72 \mathrm{~h}$, the cell viability of siSIL1 transfected U251 cells was significantly decreased compared to that of siNC transfected cells $(P<0.05)$. The inhibitory effect of siSIL1 knockdown on U251 cell proliferation was also demonstrated in clone formation assay (Figure 2D). The average clone number was decreased from $152 \pm 16$ of the siNC group to $33 \pm 8$ of the siSIL1 group.

The effect of SIL1 downregulation on U251 cell migration was also detected by using a transwell migration assay. As shown in Figure 2E, the migrated cells (crystal violet stained) in siSIL1 transfected wells were significantly decreased compared to those in the siNC group. The quantified result of cell migration is shown in Figure $2 \mathrm{~F}$, which indicated that the average migrated cell number per field was reduced from $250 \pm 24$ of the siNC group to $85 \pm 12$ of the siSIL1 group, with the inhibitory rate reaching $69 \%$.

In summary, siSIL1 transfection significantly inhibited U251 cell proliferation and migration, which suggested that SIL1 played an oncogenic role in glioma.

\section{Downregulation of SILI induced apoptosis without affecting cell cycle}

In order to determine what factors lead to the inhibition of cell proliferation, we performed flow cytometry to analyze cell cycle and apoptosis of U251 cells transfected with siSIL1. The cell populations in each phase of cell cycle are shown in Figure $3 \mathrm{~A}$ and B. It was suggested that there was no significant difference of cell population between siNC and siSIL1 group. The result suggested that siSIL1 transfection did not affect U251 cell cycle progression. However, we found that siSIL1 transfection significantly increased cell apoptosis percentage in U251 cells, from $8.78 \%$ of siNC group to $12.92 \%$ of siSIL1 group (Figure $3 \mathrm{C}$ and $\mathrm{D}, P<0.05$ ). We also detected the expression of apoptosis associated proteins. As shown in Figure 3E and F, siSIL1 transfection increased the expression of pro-apoptotic protein Bax by 2.35 -fold and Caspase3-P17 by 1.7-fold. Taken together, downregulation of siSIL1 induced cell apoptosis through downregulating pro-apoptotic proteins Bax and Caspase3-P17 but did not affect $\mathrm{U} 251$ cell cycle progression.

\section{Downregulation of SILI inhibited AKT/mTOR signaling pathway}

AKT/mTOR signaling pathway is a classic cell survival pathway which is persistently activated in many cancers, including glioma. ${ }^{16}$ In order to explain the mechanism underlying the inhibitory effect of siSIL1 downregulation on U251 cells we investigated the expression of AKT/mTOR members 
A

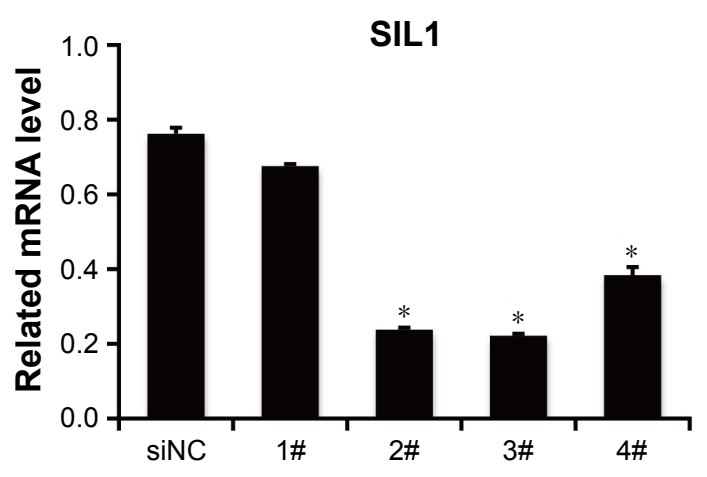

C

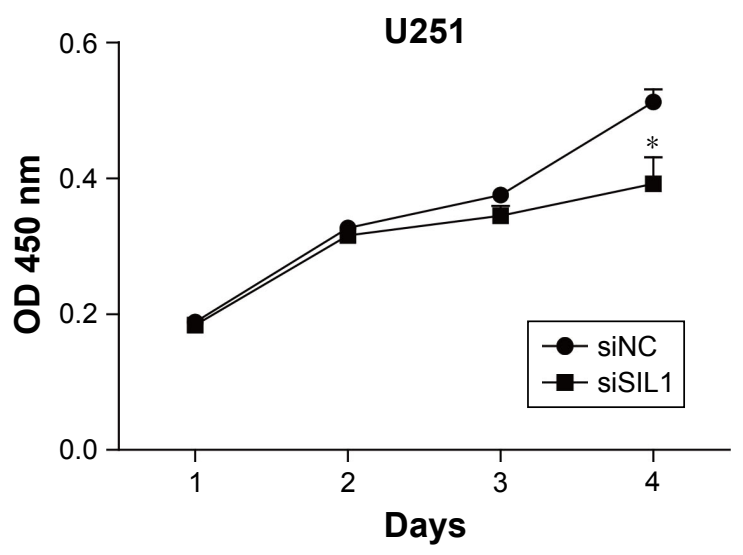

B

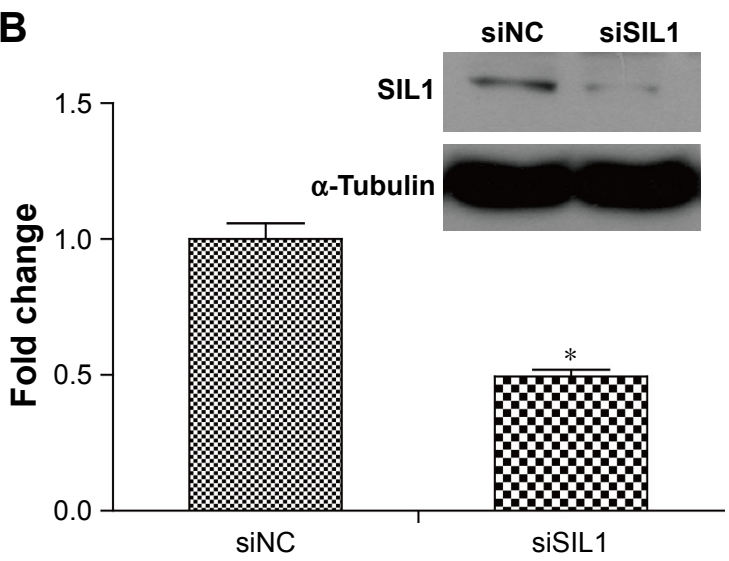

D

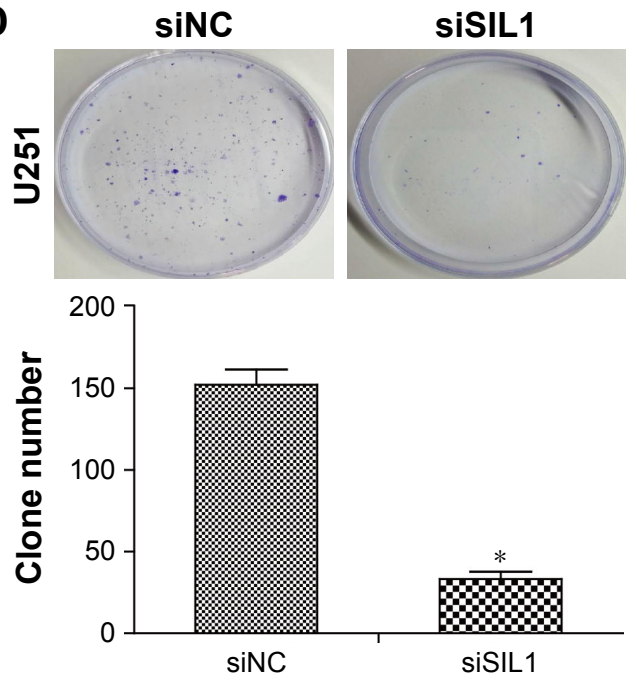

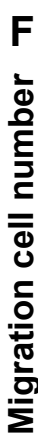

U251
ㅎํ

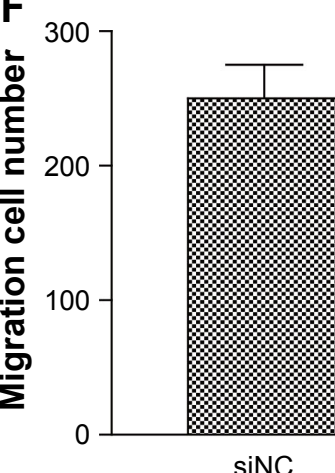

siSIL1
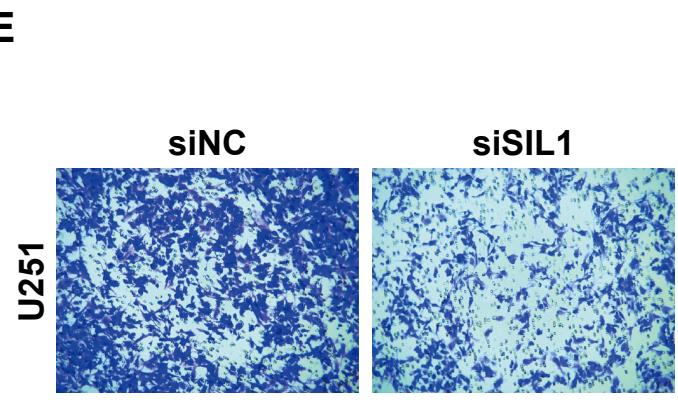

E

Figure 2 Downregulation of SILI inhibited cell proliferation and migration in U25I cells. (A) SILI expression was efficiently downregulated by siSILI transfection. qPCR demonstrated that siRNA 2, 3, and 4 inhibited SILI expression efficiently. (B) Western blot verified the inhibition of SILI expression. (C) Cell proliferation detected by CCK8 assay. (D) Cell proliferation detected by clone formation assay. (E and F) Cell migration detected by transwell assay.

Note: $* P<0.05$

Abbreviations: CCK8, cell counting kit-8; qPCR, quantitative polymerase chain reaction; NC, negative control.

by using Western blot assay. AKT plays a central regulatory role in this signaling pathway and phosphorylation-activated AKT regulates many cell processes by phosphorylating a cohort of substrates, including mTOR. When phosphorylated, mTOR will be promoting cell protein synthesis by phosphorylating $\mathrm{p} 70 \mathrm{~S} 6 \mathrm{~K}$. The Western blot results presented in Figure 4 showed that p-AKT and p-mTOR levels were significantly decreased in the siSIL1 group compared to the siNC group, while the expression of AKT and mTOR was not impacted. The expression of p70S6K was significantly 


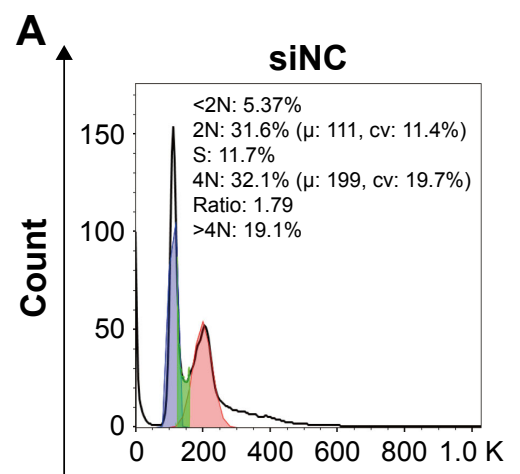

siSIL1

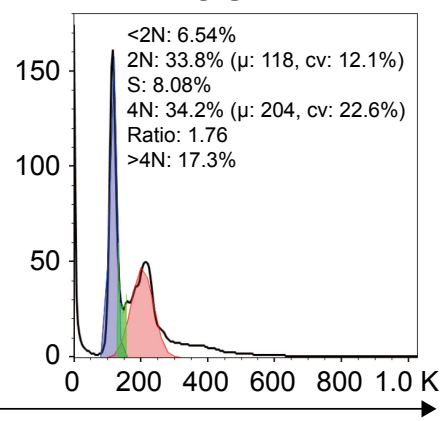

PI (DNA content)

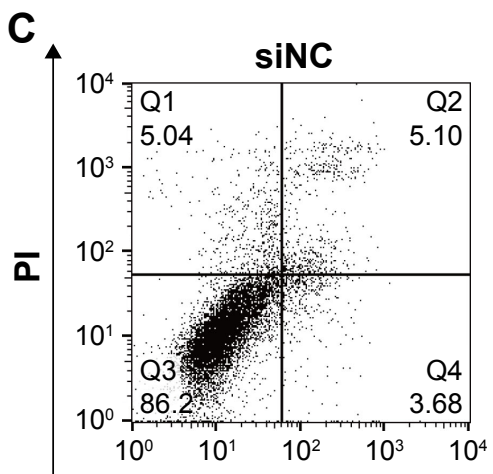

\section{Annexin V}

E

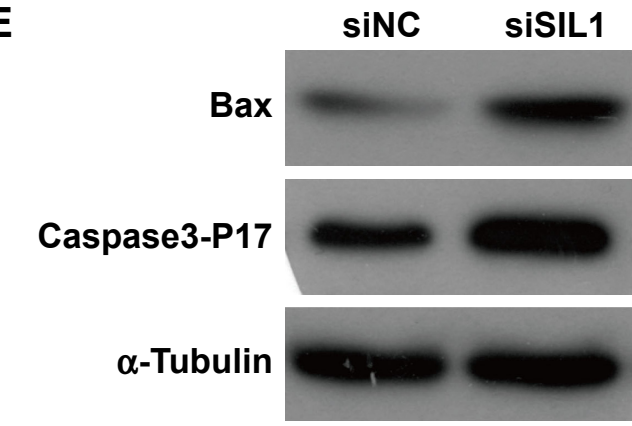

B

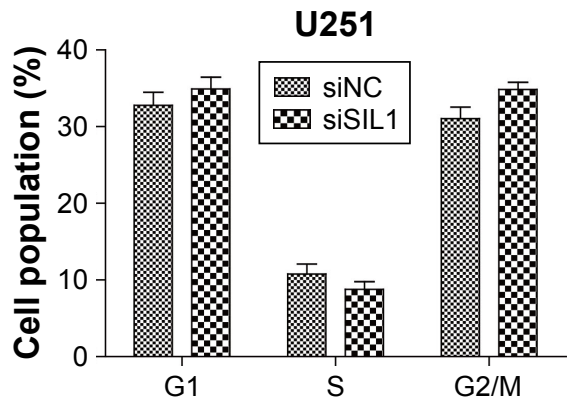

D

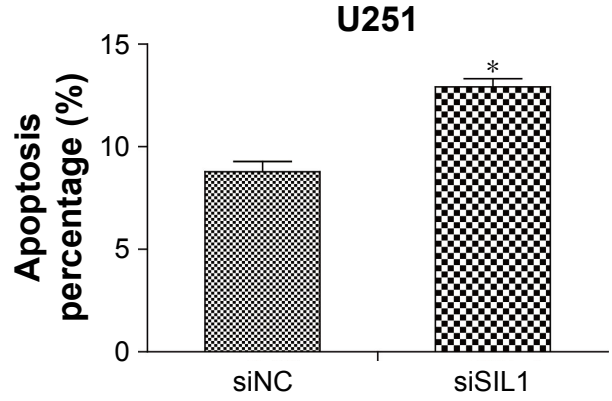

F

U251

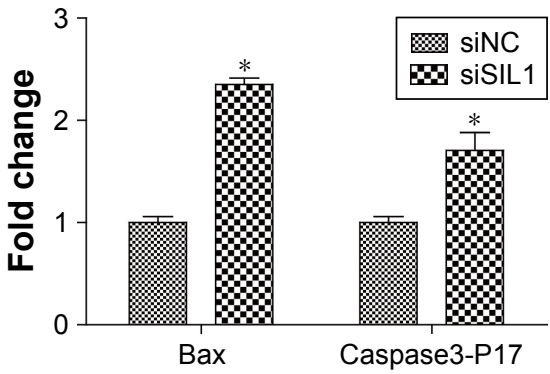

Figure 3 Downregulation of SILI induced apoptosis and the expression of pro-apoptotic genes but did not affect cell cycle in U25I cells. (A and B) U25I cell cycle detected by flow cytometry; (C and D) U25I cell apoptosis detected by flow cytometry; (E and F) expression of pro-apoptotic protein Bax and Caspase3-PI7 detected by Western blot.

Note: $* P<0.05$

Abbreviations: PI, propidium iodide; NC, negative control.

inhibited by siSIL1 transfection. These data suggested that AKT/mTOR signaling pathway was significantly inhibited in siSIL1 transfected U251 cells.

\section{Discussion}

Protein folding is one of the basic functions of cells for survival, and protein folding chaperones and cochaperones are the main functional molecules that carry out this process. $\mathrm{BiP}$ is a molecular chaperone of the HSP70 family that is localized to the ER. BiP binds new-synthesized proteins and translocates them into ER. ${ }^{5}$ In addition, BiP also binds to those abnormally folded proteins destined to be degraded and transfers them out of the ER. ${ }^{5}$ BiP can also be activated by ER stress and upregulated when unfolded protein accumulation. ${ }^{5,17}$ This property provides a marker of the disease state caused by the misfolding of secreted and transmembrane proteins. ${ }^{5,17} \mathrm{BiP}$ function requires the hydrolysis of ATP to provide energy, and in this process SIL1 functions as a nucleic acid exchange factor to release ADP from BiP. ${ }^{5,18}$ SIL1 is ubiquitously expressed in all human tissues. However, SIL1 deletion studies find that function loss of SIL1 only leads to vulnerability of skeletal muscle 

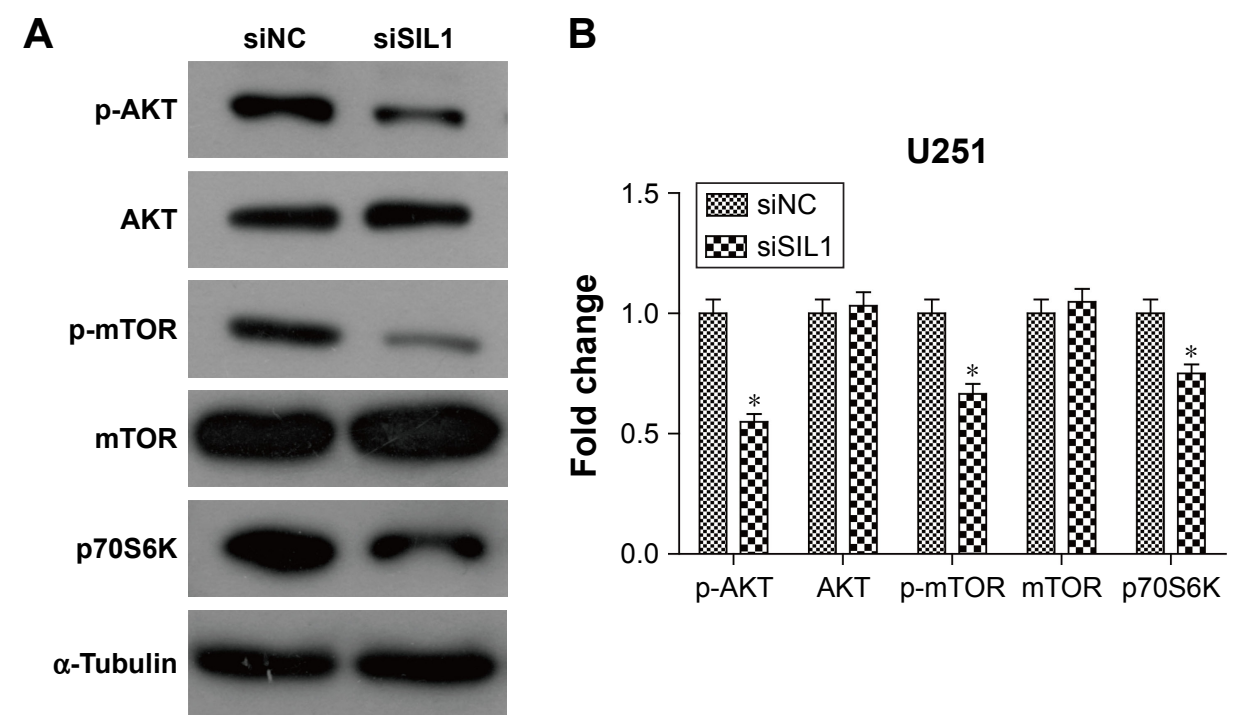

Figure 4 Downregulation of SILI inhibited AKT/mTOR signaling pathway. (A) Western blot image and (B) quantification analysis indicated that siSILI reduced the phosphorylation level of AKT and mTOR without affecting protein expression, as well as decreasing expression of the downstream effector p70S6K. Protein expression was normalized to siNC group.

Note: $* P<0.05$

and central nervous system in humans, eg, in MSS, ${ }^{7,19-21}$ and SIL1 overexpression was demonstrated a beneficial effect in surviving neurons in neurodegenerative disorders such as ALS and AD. ${ }^{9,12}$ Other studies provide some new knowledge of SIL1 function. For instance, Inaguma et al reveal that SIL1 plays an indispensable role in the structural construction of the developing cerebral cortex. ${ }^{22}$ Ittner et al report that SIL1 is required for islet insulin content, islet sizing, glucose tolerance, and glucose-stimulated insulin secretion in vivo. ${ }^{23}$ Ichhaporia et al report that SIL1 is dispensable for antibody assembly or secretion. ${ }^{24}$ However, studies of the function of SIL1 in tumor, especially nervous system neoplasms, remain scarce. In our research, we investigate the functions of SIL1 by knocking down gene expression in U251 cells.

At the beginning of the study, we searched for the expression of SIL1 in all 31 tumor types on GEPIA and found that SIL1 expression exhibited significant difference in 6 tumor types compared with normal controls, including lymphoid neoplasm diffuse large B-cell lymphoma (DLBC), GBM, LGG, prostate adenocarcinoma (PRAD), testicular germ cell tumors (TGCT), thymoma (THYM). Except for a significant decrease in PRAD, SIL1 was upregulated in all the other tumors. The survival plot of GBM and LGG suggested that patients with high SIL1 expression tended to have a poor survival. Later IHC results also demonstrated that SIL1 expression in glioma tissues significantly increased compared to paracarcinoma tissues $(P<0.05)$. So SIL1 might be a poor prognostic gene and involved in glioma progression. Consequently, we constructed a siSIL1 to downregulate the expression of SIL1 in U251 cells and examine the effects.
It was found that, compared to siNC, siSIL1 significantly inhibited U251 cell proliferation and migration. Flow cytometry detection demonstrated that siSIL1 induced apoptosis of U251 cell but did not affect the cell cycle. Moreover, pro-apoptosis proteins Bax and Caspase3-P17 were found to be upregulated by siSIL1. The mechanism study demonstrated that SIL1 knockdown could inhibit phosphorylation level of both AKT and mTOR without impacting their protein expression, which represented an inactivated state of the AKT/mTOR pathway. The downstream effector protein p70S6K was also downregulated by siSIL1. AKT/ mTOR signaling pathway is activated very frequently in human cancers through a series of genetic and epigenetic regulation. The signaling pathway is reported to promote many cancer properties such as cell survival, growth, and proliferation. ${ }^{25,26}$

\section{Conclusion}

For the first time we report that SIL1 plays an oncogenic function by AKT/mTOR signaling pathway in glioma cells. Our work provides a new insight into SIL1 in glioma progression, which may contribute to the treatment of glioma in the future.

\section{Disclosure}

The authors report no conflicts of interest in this work.

\section{References}

1. Gabriel A, Batey J, Capogreco J, et al. Adult brain cancer in the U.S. black population: a Surveillance, Epidemiology, and End Results (SEER) analysis of incidence, survival, and trends. Med Sci Monit. 2014;20:1510-1517. 
2. Avni D, Glucksam Y, Zor T. The phosphatidylinositol 3-kinase (PI3K) inhibitor LY294002 modulates cytokine expression in macrophages via p50 nuclear factor $\mathrm{kB}$ inhibition, in a PI3K-independent mechanism. Biochem Pharmacol. 2012;83(1):106-114.

3. Yang Z, Xie Q, Hu CL, et al. CHL1 is expressed and functions as a malignancy promoter in glioma cells. Front Mol Neurosci. 2017;10:324.

4. Jemal A, Siegel R, Xu J, Ward E. Cancer statistics. CA Cancer J Clin. 2010;60(5):277-300.

5. Gething MJ. Role and regulation of the ER chaperone BiP. Semin Cell Dev Biol. 1999;10(5):465-472.

6. Chung KT, Shen Y, Hendershot LM. BAP, a mammalian BiP-associated protein, is a nucleotide exchange factor that regulates the ATPase activity of BiP. J Biol Chem. 2002;277(49):47557-47563.

7. Zhao L, Longo-Guess C, Harris BS, Lee JW, Ackerman SL. Protein accumulation and neurodegeneration in the woozy mutant mouse is caused by disruption of SIL1, a cochaperone of BiP. Nat Genet. 2005;37(9): 974-979.

8. Siegenthaler KD, Pareja KA, Wang J, Sevier CS. An unexpected role for the yeast nucleotide exchange factor Sill as a reductant acting on the molecular chaperone BiP. Elife. 2017;6:e24141.

9. Liu ZC, Chu J, Lin L, et al. SIL1 Rescued Bip elevation-related tau hyperphosphorylation in ER stress. Mol Neurobiol. 2016;53(2):983-994.

10. Labisch T, Buchkremer S, Phan V, et al. Tracking effects of SIL1 increase: taking a closer look beyond the consequences of elevated expression level. Mol Neurobiol. 2018;55(3):2524-2546.

11. Howes J, Shimizu Y, Feige MJ, Hendershot LM. C-terminal mutations destabilize SIL1/BAP and can cause Marinesco-Sjogren syndrome. J Biol Chem. 2012;287(11):8552-8560.

12. Filezac de L'Etang A, Maharjan N, Cordeiro Brana M, et al. MarinescoSjogren syndrome protein SIL1 regulates motor neuron subtypeselective ER stress in ALS. Nat Neurosci. 2015;18(2):227-238.

13. Karim MA, Parsian AJ, Cleves MA, et al. A novel mutation in BAP/ SIL1 gene causes Marinesco-Sjogren syndrome in an extended pedigree. Clin Genet. 2006;70(5):420-423.

14. Camp RL, Dolled-Filhart M, Rimm DL. X-tile: a new bio-informatics tool for biomarker assessment and outcome-based cut-point optimization. Clin Cancer Res. 2004;10(21):7252-7259.
15. Tang Z, Li C, Kang B, Gao G, Li C, Zhang Z. GEPIA: a web server for cancer and normal gene expression profiling and interactive analyses. Nucleic Acids Res. 2017;45(W1):W98-W102.

16. Dasari VR, Kaur K, Velpula KK, et al. Upregulation of PTEN in glioma cells by cord blood mesenchymal stem cells inhibits migration via downregulation of the PI3K/Akt pathway. PLoS One. 2010;5(4):e10350.

17. Ranganathan AC, Zhang L, Adam AP, Aguirre-Ghiso JA. Functional coupling of p38-induced up-regulation of $\mathrm{BiP}$ and activation of RNAdependent protein kinase-like endoplasmic reticulum kinase to drug resistance of dormant carcinoma cells. Cancer Res. 2006;66(3):1702-1711.

18. Dudek J, Benedix J, Cappel S, et al. Functions and pathologies of BiP and its interaction partners. Cell Mol Life Sci. 2009;66(9):1556-1569.

19. Anttonen AK, Mahjneh I, Hamalainen RH, et al. The gene disrupted in Marinesco-Sjogren syndrome encodes SIL1, an HSPA5 cochaperone. Nat Genet. 2005;37(12):1309-1311.

20. Senderek J, Krieger M, Stendel C, et al. Mutations in SIL1 cause Marinesco-Sjogren syndrome, a cerebellar ataxia with cataract and myopathy. Nat Genet. 2005;37(12):1312-1314.

21. Kollipara L, Buchkremer S, Coraspe JAG, et al. In-depth phenotyping of lymphoblastoid cells suggests selective cellular vulnerability in Marinesco-Sjogren syndrome. Oncotarget. 2017;8(40):68493-68516.

22. Inaguma $\mathrm{Y}$, Hamada $\mathrm{N}$, Tabata $\mathrm{H}$, et al. SIL1, a causative cochaperone gene of Marinesco-Sojgren syndrome, plays an essential role in establishing the architecture of the developing cerebral cortex. EMBO Mol Med. 2014;6(3):414-429.

23. Ittner AA, Bertz J, Chan TY, van Eersel J, Polly P, Ittner LM. The nucleotide exchange factor SIL1 is required for glucose-stimulated insulin secretion from mouse pancreatic beta cells in vivo. Diabetologia. 2014;57(7):1410-1419.

24. Ichhaporia VP, Sanford T, Howes J, Marion TN, Hendershot LM Sil1, a nucleotide exchange factor for $\mathrm{BiP}$, is not required for antibody assembly or secretion. Mol Biol Cell. 2015;26(3):420-429.

25. Tavares MR, Pavan IC, Amaral CL, Meneguello L, Luchessi AD, Simabuco FM. The S6K protein family in health and disease. Life Sci. 2015;131:1-10.

26. Arcaro A. Targeting PI3K/mTOR Signaling in Cancer. Front Oncol. 2014;4:84.
OncoTargets and Therapy

\section{Publish your work in this journal}

OncoTargets and Therapy is an international, peer-reviewed, open access journal focusing on the pathological basis of all cancers, potential targets for therapy and treatment protocols employed to improve the management of cancer patients. The journal also focuses on the impact of management programs and new therapeutic agents and protocols on

\section{Dovepress}

patient perspectives such as quality of life, adherence and satisfaction. The manuscript management system is completely online and includes a very quick and fair peer-review system, which is all easy to use. Visit http://www.dovepress.com/testimonials.php to read real quotes from published authors. 